\title{
Families of Graphs Closed Under Taking Powers*
}

\author{
Mingjang Chen ${ }^{1}$ and Gerard J. Chang ${ }^{2}$ \\ Department of Applied Mathematics, National Chiao Tung University. Hsinchu 30050, \\ Taiwan, R.O.C. ${ }^{1}$ e-mail: mjchen@cc.nctu.edu.tw; ${ }^{2}$ e-mail: gjchang@math.nctu.edu.tw
}

\begin{abstract}
This paper gives simple proofs for " $G^{k} \in \mathscr{A}$ implies $G^{k+1} \in \mathscr{A}$ " when $\mathscr{A}$ is the family of all interval graphs, all proper interval graphs, all cocomparability graphs, or all $m$-trapezoid graphs.
\end{abstract}

\section{Introduction}

In a graph $G=(V, E)$, the distance $d_{G}(x, y)$ between two vertices $x$ and $y$ is the minimum number of edges in an $x-y$ path; $d_{G}(x, y)=\infty$ if there exists no $x-y$ path. For a positive integer $k$, the kth power of a graph $G=(V, E)$ is the graph $G^{k}=\left(V, E^{k}\right)$ whose vertex set is $V$ and edge set $E^{k}=\left\{x y: 1 \leq d_{G}(x, y) \leq k\right\}$.

Powers of graphs have been studied from different points of view. For instance, researchers are interested in knowing which families of graphs are closed under taking powers. Well-known families of this kind are interval graphs, proper interval graphs, strongly chordal graphs, circular-arc graphs, cocomparability graphs among others. A more general question is, for a family $\mathscr{A}$ of graphs, whether $G^{k} \in \mathscr{A}$ implies $G^{k+1} \in \mathscr{A}$.

The first surprising result in this line was given by Lubiw [18], who proved that powers of strongly chordal graphs are strongly chordal. Hoffman et al. [14] gave a simple proof of this result. Knowing a similar result is impossible for chordal graphs, Chang and Nemhauser [3] showed that if $G$ and $G^{2}$ are chordal then so are all powers of $G$. On the other hand, Balakirishnan and Paulraja [2] proved that odd powers of chordal graphs are chordal. An even more interesting result, with an elegant proof, was given by Duchet [10] that if $G^{k}$ is chordal then so is $G^{k+2}$.

Since then, many authors worked on the problem "whether $G^{k} \in \mathscr{A}$ implies $G^{k+1} \in \mathscr{A}^{\prime}$ for various families $\mathscr{A}$. Typical examples are strongly chordal graphs [21], interval graphs [20], proper interval graphs [20], $m$-trapezoid graphs [11], cocomparability graphs [11]. Most of them are proved in some clever, but slightly complicated ways. The main effort of this paper is to give simple proofs for

\footnotetext{
* Supported in part by the National Science Council under grant NSC87-2115-M-009-007
} 
interval graphs, proper interval graphs, cocomparability graphs, and $m$-trapezoid graphs by a "vertex ordering" methodology.

\section{Powers of Graphs}

The concept of intersection graphs plays an important role in graph theory. The intersection graph of a family $\mathscr{F}$ of sets is the graph whose vertices have a one-to-one correspondence to the sets in $\mathscr{F}$, and two distinct vertices are adjacent if and only if their corresponding sets intersect. In this definition, $\mathscr{F}$ is called an intersection model of its intersection graph. It is an easy exercise to show that any graph is the intersection graph of some family of sets. However, if the sets in $\mathscr{F}$ have special structures, then its intersection graph is usually well-behaved. Recently intersection graphs of the following objects have been studied extensively by many authors: intervals on the real line, boxes (balls) in the $n$-dimensional Euclidean space, arcs in a circle, trapezoids between two parallel lines on a plane, to name a few.

Among these, interval graphs, which are intersection graphs of intervals on the real line, have been most extensively studied not only because they are wellbehaved, but also because they are applicable to many fields such as biology and computer science, e.g., see [24]. For studying the domination problem, Ramalingam and Pandu Rangan [19] gave that a graph $G$ is an interval graph if and only if it has an interval ordering, which is an ordering of $V(G)$ into $\left[v_{1}, v_{2}, \ldots, v_{n}\right]$ such that

$$
i<\ell<j \quad \text { and } \quad v_{i} v_{j} \in E(G) \text { imply } v_{\ell} v_{j} \in E(G)
$$

This can be seen by sorting the right endpoints of intervals correspondent to the vertices of the interval graph. Using this, we now give an alternative proof for Raychaudhuri's [20] result on interval graphs.

Theorem 1. Suppose $G$ is a graph and $k$ a positive integer. If $G^{k}$ is an interval graph, then so is $G^{k+1}$.

Proof. Let $\left[v_{1}, v_{2}, \ldots, v_{n}\right]$ be an interval ordering of $G^{k}$. Consider $G^{k+1}$ and the ordering $\left[v_{1}, v_{2}, \ldots, v_{n}\right]$. Suppose $i<\ell<j$ and $v_{i} v_{j} \in E\left(G^{k+1}\right)$, i.e., $d_{G}\left(v_{i}, v_{j}\right) \leq$ $k+1$. If $d_{G}\left(v_{i}, v_{j}\right) \leq k$, then $v_{i} v_{j} \in E\left(G^{k}\right)$ and so $v_{\ell} v_{j} \in E\left(G^{k}\right) \subseteq E\left(G^{k+1}\right)$. Now, suppose $d_{G}\left(v_{i}, v_{j}\right)=k+1$. Let $P$ be a shortest $v_{i}-v_{j}$ path in $G$ and let $v_{a}$ be the vertex adjacent to $v_{j}$ on $P$. Then, $d_{G}\left(v_{i}, v_{a}\right)=k$ and $d_{G}\left(v_{a}, v_{j}\right)=1$. So, $v_{i} v_{a} \in E\left(G^{k}\right)$ and $v_{a} v_{j} \in E\left(G^{k}\right)$. If $i<\ell<a$, then $v_{\ell} v_{a} \in E\left(G^{k}\right)$ and so $d_{G}\left(v_{\ell}, v_{j}\right) \leq d_{G}\left(v_{\ell}, v_{a}\right)+$ $d_{G}\left(v_{a}, v_{j}\right) \leq k+1$. If $a<\ell<j$, then $v_{\ell} v_{j} \in E\left(G^{k}\right) \subseteq E\left(G^{k+1}\right)$. Therefore, $v_{\ell} v_{j} \in E$ $\left(G^{k+1}\right)$ in any case. Hence, $\left[v_{1}, v_{2}, \ldots, v_{n}\right]$ is an interval ordering of $G^{k+1}$ and $G^{k+1}$ is an interval graph.

Corollary 2. Powers of interval graphs are interval graphs.

A proper interval graph is an interval graph with an interval model in which no interval is a proper subset of another interval. Ding [9] and Roberts [23] proved 
that a graph is a proper interval graph if and only if it has an proper interval ordering, which is an ordering of its vertex set into $\left[v_{1}, v_{2}, \ldots, v_{n}\right]$ such that $\left[v_{1}, v_{2}, \ldots, v_{n}\right]$ and $\left[v_{n}, v_{n-1}, \ldots, v_{1}\right]$ are interval orderings, or equivalently,

$$
i<\ell<j \text { and } v_{i} v_{j} \in E(G) \text { imply } v_{i} v_{\ell} \in E(G) \text { and } v_{\ell} v_{j} \in E(G) \text {. }
$$

Using this, we have The following simple proof for Raychaudhuri's [20] result on proper interval graphs.

Theorem 3. Suppose $G$ is a graph and $k$ a positive integer. If $G^{k}$ is a proper interval graph, then so is $G^{k+1}$.

Proof. Let $\left[v_{1}, v_{2}, \ldots, v_{n}\right]$ be a proper interval ordering of $G^{k}$, i.e., both $\left[v_{1}, v_{2}, \ldots, v_{n}\right]$ and $\left[v_{n}, v_{n+1}, \ldots, v_{1}\right]$ are interval orderings of $G^{k}$. By the same arguments used in the proof of Theorem 1, we have that both $\left[v_{1}, v_{2}, \ldots, v_{n}\right]$ and $\left[v_{n}, v_{n-1}, \ldots, v_{1}\right]$ are interval orderings of $G^{k+1}$. Hence, $G^{k+1}$ is a proper interval graph.

Corollary 4. Powers of proper interval graphs are proper interval graphs.

A comparability graph is the underlying graph of an acyclic transitive digraph, which can be viewed as a poset. In other words, a graph $G$ is comparability if it has a transitive ordering that is an ordering of $V(G)$ into $\left[v_{1}, v_{2}, \ldots, v_{n}\right]$ such that

$$
i<\ell<j \text { and } v_{i} v_{\ell}, v_{\ell} v_{j} \in E(G) \text { imply } v_{i} v_{j} \in E(G) \text {. }
$$

A cocomparability graph is the complement of a comparability graph, i.e., it has a cocomparability ordering that is an ordering of its vertex set into $\left[v_{1}, v_{2}, \ldots, v_{n}\right]$ such that

$$
i<\ell<j \text { and } v_{i} v_{j} \in E(G) \text { imply } v_{i} v_{\ell} \in E(G) \text { or } v_{\ell} v_{j} \in E(G) \text {. }
$$

Cocomparability graphs include interval graphs and $m$-trapezoid graphs defined below. Flotow [11] proved the following result for cocomparability graphs by means of $m$-trapezoid graphs. We now give a simple and direct proof.

Theorem 5. Suppose $G$ is a graph and $k$ a positive integer. If $G^{k}$ is a cocomparability graph, then so is $G^{k+1}$.

Proof. Let $\left[v_{1}, v_{2}, \ldots, v_{n}\right]$ be a cocomparability ordering of $G^{k}$. Consider $G^{k+1}$ and the ordering $\left[v_{1}, v_{2}, \ldots, v_{n}\right]$. Suppose $i<\ell<j$ and $v_{i} v_{j} \in E\left(G^{k+1}\right)$, i.e., $d_{G}\left(v_{i}, v_{j}\right) \leq k+1$. If $d_{G}\left(v_{i}, v_{j}\right) \leq k$, then $v_{i} v_{j} \in E\left(G^{k}\right)$, which implies that either $v_{i} v_{\ell} \in E\left(G^{k}\right) \subseteq E\left(G^{k+1}\right)$ or $v_{\ell} v_{j} \in E\left(G^{k}\right) \subseteq E\left(G^{k+1}\right)$. Now, suppose $d_{G}\left(v_{i}, v_{j}\right)=$ $k+1$. Choose a vertex $v_{a}$ such that $d_{G}\left(v_{i}, v_{a}\right)=k$ and $d_{G}\left(v_{a}, v_{j}\right)=1$. Then, $v_{i} v_{a} \in E\left(G^{k}\right)$ and $v_{a} v_{j} \in E\left(G^{k}\right)$. If $i<\ell<a$, then either $d_{G}\left(v_{i}, v_{\ell}\right) \leq k$ or $d_{G}\left(v_{\ell}, v_{a}\right) \leq k$. For the former case, $v_{i} v_{\ell} \in E\left(G^{k}\right) \subseteq E\left(G^{k+1}\right)$; for the latter case, $d_{G}\left(v_{\ell}, v_{j}\right) \leq d_{G}\left(v_{\ell}, v_{a}\right)+d_{G}\left(v_{a}, v_{j}\right) \leq k+1$ and so $v_{\ell} v_{j} \in E\left(G^{k+1}\right)$. If $a<\ell<j$, then 
either $d_{G}\left(v_{a}, v_{\ell}\right) \leq k \quad$ or $\quad d_{G}\left(v_{\ell}, v_{j}\right) \leq k$. For the former case, $d_{G}\left(v_{\ell}, v_{j}\right) \leq d_{G}\left(v_{a}, v_{\ell}\right)+d_{G}\left(v_{a}, v_{j}\right) \leq k+1$ and so $v_{\ell} v_{j} \in E\left(G^{k+1}\right)$; for the latter case, $v_{\ell} v_{j} \in E\left(G^{k}\right) \subseteq E\left(G^{k+1}\right)$. Hence, in any case, $\left[v_{1}, v_{2}, \ldots, v_{n}\right]$ is a cocomparability ordering of $G^{k+1}$ and $G^{k+1}$ is a cocomparability graph.

Corollary 6. Powers of cocomparability graphs are cocomparability.

Although, the result for cocomparability graphs can be proved without using $m$-trapezoid graphs, the result for $m$-trapezoid graphs has its own interest. As the final part of this paper, we also give a new proof for the result on $m$-trapezoid graphs.

Suppose $m \geq 0$ and $L_{0}, L_{1}, \ldots, L_{m}$ are $m+1$ parallel lines, indexed to their ordering, in the plane. Suppose $\left[a_{i}, b_{i}\right]$ is an interval in $L_{i}$ for $0 \leq i \leq m$. These intervals define an m-trapezoid that is the region bounded by the polygon $a_{0}, a_{1}, \ldots, a_{m}, b_{m}, b_{m-1}, \ldots, b_{0}, a_{0}$. An m-trapezoid graph is the intersection graph of $m$-trapezoids over $m+1$ parallel lines in the plane. Without loss of generality, we may assume that all right endpoints $b_{i}$ 's for different $m$-trapezoids are distinct. Note that 0 -trapezoid graphs are precisely interval graphs; 1-trapezoid graphs are the usual trapezoid graphs, which include permutation graphs; and $m$-trapezoid are precisely comparability graphs of posets with interval dimension at most $m+1$ (see $[11,25])$.

Lemma 7. A graph $G=(V, E)$ is an m-trapezoid graph if and only if it has a family of $m$-trapezoid orderings that is a set $\left\{<_{0},<_{1}, \ldots,<_{m}\right\}$ of $m+1$ orderings of $V$ such that the following two conditions hold for all vertices $x$ and $y$.

$(\mathrm{T} 1(x, y, G))$ If $x$ and $y$ disagree in two orderings $<_{i}$ and $<_{j}$ (i.e., $x<_{i} y$ but $y<_{j} x$ or $x<_{j} y$ but $\left.y<_{i} x\right)$, then $x y \in E$.

$(\mathrm{T} 2(x, y, G))$ If $x$ and $y$ agree in all orderings (say, $x<_{\ell} y$ for all $\left.\ell\right)$ and $x y \in E$, then there exists some $i^{*}$ such that $x<_{i^{*}} z<_{i^{*}} y$ implies $z y \in E$.

Proof. $(\Rightarrow)$ Suppose $G$ is an $m$-trapezoid graph whose $m$-trapezoid representation is over the parallel lines $L_{0}, L_{1}, \ldots, L_{m}$. For each vertex $v \in V$, let $\left[a_{i}^{v}, b_{i}^{v}\right]$ be the interval for the $m$-trapezoid of $v$ in $L_{i}$. Define an ordering $<_{i}$ of $V$ by

$$
x<_{i} y \text { if and only if } b_{i}^{x}<b_{i}^{y} .
$$

It is straightford to check that the two conditions (T1) and (T2) hold.

$(\Leftarrow)$ Conversely, suppose $G$ has a family of $m$-trapezoid orderings $\left\{<_{0},<_{1}, \ldots,<_{m}\right\}$. Construct $m+1$ parallel lines $L_{0}, L_{1}, \ldots, L_{m}$. For any vertex $v \in V$ and any line $L_{i}$, choose $b_{i}^{v}$ such that (2.1) holds. Define

$$
a_{i}^{v}=\min \left(\left\{b_{i}^{v}\right\} \cup\left\{b_{i}^{x}: x v \in E, x<_{i} v \text {, and } z v \in E \text { whenever } x<_{i} z<_{i} v\right\}\right) \text {. }
$$

Then the $|V| m$-trapezoids defined by the intervals $\left[a_{i}^{v}, b_{i}^{v}\right]$ determine an $m$-trapezoid graph, which can be verified to be the graph $G$. 
Theorem 8. Suppose $G$ is a graph and $k$ a positive integer. If $G^{k}$ is an $m$-trapezoid graph, then so is $G^{k+1}$.

Proof. Let $\left\{<_{0},<_{1}, \ldots,<_{m}\right\}$ be a family of $m$-trapezoid orderings of $G^{k}$. Consider $G^{k+1}$ and the family $\left\{<_{0},<_{1}, \ldots,<_{m}\right\}$. Since (T1 $\left.\left(x, y, G^{k}\right)\right)$ holds for all $x$ and $y$ and $E\left(G^{k}\right) \subseteq E\left(G^{k+1}\right),\left(\mathrm{T} 1\left(x, y, G^{k+1}\right)\right)$ holds for all $x$ and $y$. For (T2 $\left.\left(x, y, G^{k+1}\right)\right)$, suppose $x<_{\ell} y$ for all $\ell$ and $x y \in E\left(G^{k+1}\right)$. Choose a vertex $w$ such that $d_{G}(x, w) \leq k$ and $d_{G}(w, y)=1$.

Note that either $w<_{i} x$ for some $i$ or $x<_{\ell} w$ for all $\ell$. For the former case, choose $i^{*}=i$. For the later case, (T2 $\left.\left(x, w, G^{k}\right)\right)$ holds for some $<_{i_{1}^{*}}$ and we choose $i^{*}=i_{1}^{*}$. In either case, consider any $z$ with $x<_{i^{*}} z<_{i^{*}} y$. If $z$ and $y$ disagree in two orderings, then $\left(\mathrm{T} 1\left(z, y, G^{k}\right)\right)$ implies $z y \in E\left(G^{k}\right) \subseteq E\left(G^{k+1}\right)$. So we may assume that $z$ and $y$ agree in all orderings. If $z$ and $w$ disagree in two orderings, then $\left(\mathrm{T} 1\left(z, w, G^{k}\right)\right)$ implies $z w \in E\left(G^{k}\right)$ and so $z y \in E\left(G^{k+1}\right)$. So we may assume that $z$ and $w$ agree in all orderings.

Case 1. $w<_{i} x$ for some $i$ and $i^{*}=i$.

As $w<_{i} x<_{i} z<_{i} y$, we have $w<_{\ell} z<_{\ell} y$ for all $\ell$. Since $w y \in E(G) \subseteq E\left(G^{k}\right)$, (T2 $\left.\left(w, y, G^{k}\right)\right)$ holds for some $i_{2}^{*}$. In this case, $z y \in E\left(G^{k}\right) \subseteq E\left(G^{k+1}\right)$.

Case 2. $x<_{\ell} w$ for all $\ell$ and $i^{*}=i_{1}^{*}$.

In this case, $x<_{i_{1}^{*}} z<_{i_{1}^{*}} w$ or $z=w$ or $w<_{i_{1}^{*}} z<_{i_{1}^{*}} y$. For the case of $x<_{i_{1}^{*}} z<_{i_{1}^{*}} w$, (T2 $\left(x, w, G^{k}\right)$ ) implies $z w \in E\left(G^{k}\right)$ and so $z y \in E\left(G^{k+1}\right)$. For the case of $z=w$, $z y=w y \in E(G) \subseteq E\left(G^{k+1}\right)$. For the case of $w<_{i_{1}^{*}} z<_{i_{1}^{*}} y$, we have $w<_{\ell} z<_{\ell} y$ for all $\ell$ and so (T2 $\left.\left(w, y, G^{k}\right)\right)$ holds for some $i_{2}^{*}$, which implies $z y \in E\left(G^{k}\right) \subseteq E\left(G^{k+1}\right)$.

In any case, $\left(\mathrm{T} 2\left(x, y, G^{k+1}\right)\right)$ holds. Hence $\left\{<_{0},<_{1}, \ldots,<_{m}\right\}$ is a family of $m$ trapezoid orderings for $G^{k+1}$ and $G^{k+1}$ is an $m$-trapezoid graph.

Corollary 9. Powers of trapezoid graphs are trapezoid graphs.

In fact, Theorems 1 and 5 also follow from Theorem 8 .

Acknowledgments. The authors thank the referees for many constructive suggestions.

\section{References}

1. Balakrishnan, R., Paulraja, P.: Graphs whose squares are chordal. Indian J. Pure Appl. Math. 12, 193-194 (1981); with erratum same J. 12, 1062 (1981)

2. Balakrishnan, R., Paulraja, P.: Powers of chordal graphs. J. Aust. Math. Soc. Ser. A 35, 211-217 (1983)

3. Chang, G.J., Nemhauser, G.L.: The $k$-domination and $k$-stability problems on sun-free chordal graphs. SIAM J. Algebraic Discrete Methods 5, 332-345 (1984)

4. Chang, G.J., Nemhauser, G.L.: Covering, packing and generalized perfection. SIAM J. Algebraic Discrete Methods 6, 109-132 (1985)

5. Chen, C.Y., Chang, C.C., Chang, G.J.: Proper interval graphs and the guard problem. Discrete Math. 170, 223-230 (1997)

6. Dagan, I., Golumbic, M.C., Pinter, R.Y.: Trapezoid graphs and their coloring. Discrete Appl. Math. 21, 35-46 (1988) 
7. Dahlhaus, E., Duchet, P.: On strongly chordal graphs. Ars Comb. 24B, 23-30 (1987)

8. Damaschke, P.: Distances in cocomparability graphs and their powers. Discrete Appl. Math. 35, 67-72 (1992)

9. Ding, G.: Covering the edges with consecutive sets. J. Graph Theory 15, 559-562 (1991)

10. Duchet, P.: Classical perfect graphs. Ann. Discrete Math. 21, 67-96 (1984)

11. Flotow, C.: On powers of $m$-trapezoid graphs. Discrete Appl. Math. 63, 187-192 (1995)

12. Flotow, C.: On powers of circular arc graphs and proper circular arc graphs. Discrete Appl. Math. 69, 199-207 (1996)

13. Flotow, C.: Graphs whose powers are chordal and graphs whose powers are interval graphs. J. Graph Theory 24, 323-330 (1997)

14. Hoffman, A.J., Kolen, A.W.J., Sakarovitch, M.: Totally-balanced and greedy matrices. SIAM J. Algebraic Discrete Methods 6, 721-730 (1985)

15. Laskar, R., Shier, D.: On chordal graphs. Congr. Numerantium 29, 579-588 (1980)

16. Laskar, R., Shier, D.: On powers and centers of chordal graphs. Discrete Appl. Math. 6, 139-147 (1983)

17. Lin, Y., Skiena, S.: Algorithms for square roots of graphs. Manuscript

18. Lubiw, A.: $\Gamma$-Free Matrices. Master Thesis, Department of Combinatorics and Optimization, University of Waterloo 1982

19. Ramalingam, G., Pandu Rangan, C.: A uniform approach to domination problems on interval graphs. Inf. Process. Lett. 27, 271-274 (1988)

20. Raychaudhuri, A.: On powers of interval and unit interval graphs. Congr. Numerantium 59, 235-242 (1987)

21. Raychaudhuri, A.: On powers of strongly chordal graphs and circular arc graphs. Ars Comb. 34, 147-160 (1992)

22. Raychaudhuri, A., Roberts, F.S.: Generalized competition graphs and their applications. In: P. Brucker and R. Pauly, Methods of Oper. Res., pp. 295-311. Anton Hain, Koningstein, West Germany 1985

23. Roberts, F.S.: On the compatibility between a graph and a simple order. J. Comb. Theory, Ser. B 11, 28-38 (1971)

24. Roberts, F.S.: Graph Theory and Its Applications to Problems of Society. CBMS-NSF Reg. Conf. Ser. Appl. Math. SIAM, 1978

25. Trotter, W.T.: Combinatorics and Partially Ordered Sets: Dimension Theory. Bartimore, MD: John Hopkins University Press 1991

Received: November 21, 1997

Final version received: October 5, 1998 